\title{
PRIMITIVE LATTICE POINTS IN STARLIKE PLANAR SETS
}

\author{
Werner Georg NowaK
}

This article is concerned with the number $B_{\mathcal{D}}(x)$ of integer points with relative prime coordinates in $\sqrt{x} \mathcal{D}$, where $x$ is a large real variable and $\mathcal{D}$ is a starlike set in the Euclidean plane. Assuming the truth of the Riemann Hypothesis, we establish an asymptotic formula for $B_{\mathcal{D}}(x)$. Applications to certain special geometric and arithmetic problems are discussed.

\section{Introduction.}

Let $\mathcal{D}$ denote a subset of $\mathbb{R}^{2}$ which is starlike with respect to the origin, i.e., if $\mathbf{u} \in \mathbb{R}^{2}$ belongs to $\mathcal{D}$, automatically $\lambda \mathbf{u} \in \mathcal{D}$ for $0<\lambda<1$. The distance function $F$ of $\mathcal{D}$ is defined by

$$
F(\mathbf{u})=\inf \left\{\tau>0: \frac{\mathbf{u}}{\tau} \in \mathcal{D}\right\}
$$

with the usual understanding that $\inf \emptyset=\infty$. Let us put $Q=F^{2}$, then $Q$ is homogeneous of degree 2 . For a large real variable $x$, we define $A_{\mathcal{D}}(x)$ as the number of lattice points of $\mathbb{Z}_{*}^{2}:=\mathbb{Z}^{2} \backslash\{(0,0)\}$ in the "blown up" domain $\sqrt{x} \mathcal{D}$, i.e.,

$$
A_{\mathcal{D}}(x)=\#\left(\sqrt{x} \mathcal{D} \cap \mathbb{Z}_{*}^{2}\right)
$$

We make the supposition that $A_{\mathcal{D}}(x)$ satisfies an asymptotic formula

$$
A_{\mathcal{D}}(x)=\sum_{r=0}^{R} c_{r} x^{\alpha_{r}}+O\left(x^{\alpha}\right),
$$

with

$$
\alpha_{0}=1>\alpha_{1}>\cdots>\alpha_{R}>\alpha, \quad \alpha<\frac{1}{2}
$$


(For a wealth of results of the form (1.1) on specific planar lattice point problems the reader may consult the monograph of Krätzel [11].)

The objective of the present paper is to study the number $B_{\mathcal{D}}(x)$ of primitive lattice points in $\sqrt{x} \mathcal{D}$, i.e.,

$$
B_{\mathcal{D}}(x)=\#\left\{\mathbf{m}=\left(m_{1}, m_{2}\right) \in \mathbb{Z}_{*}^{2}: \mathbf{m} \in \sqrt{x} \mathcal{D}, \operatorname{gcd}\left(m_{1}, m_{2}\right)=1\right\} .
$$

By Möbius inversion,

$$
B_{\mathcal{D}}(x)=\sum_{m \in \mathbb{N}} \mu(m) A_{\mathcal{D}}\left(\frac{x}{m^{2}}\right)
$$

where $\mu(m)$ denotes the Möbius function. By an elementary convolution argument, one can derive from the bound

$$
\sum_{m \leq Y} \mu(m) \ll Y \omega(Y)
$$

(see Ivić [8, p. 309]), combined with (1.1) and (1.3),

$$
B_{\mathcal{D}}(x)=\sum_{r: \alpha_{r} \geq \frac{1}{2}} \frac{c_{r}}{\zeta\left(2 \alpha_{r}\right)} x^{\alpha_{r}}+O\left(x^{1 / 2} \omega(x)\right)
$$

where

$$
\omega(x)=\exp \left(-c(\log x)^{3 / 5}(\log \log x)^{-1 / 5}\right)
$$

with $c>0$, is a factor familiar from the Prime Number Theorem. (1.4) and (1.5) contain the strongest information available to date concerning zero-free regions of the Riemann zeta-function. At the present state of art, it is not possible to reduce the exponent $\frac{1}{2}$ of $x$ in the $O$-term of (1.5). This will be evident from Lemma 1 below (with $y=1$ ), in view of the fact that the Riemann zeta-function could have zeros with real part arbitrarily close to 1 .

It is therefore natural to search for stronger estimates assuming the truth of the Riemann Hypothesis (henceforth quoted as RH).

This problem has been attacked by Moroz [15], for the slightly simplified case that $R=0$. He obtained the result that

$$
B_{\mathcal{D}}(x)=c_{0} \frac{6}{\pi^{2}} x+O\left(x^{\frac{2-\alpha}{5-4 \alpha}+\varepsilon}\right) \quad(\varepsilon>0),
$$

conditionally under $\mathrm{RH}$.

We remark that recently Hensley [5] has recently written a paper on the subject, too, apparently unaware of Moroz's work. He used a methodically original approach but failed to sharpen the estimate.

In this paper, our ultimate goal will be to prove the following. 
Theorem. Suppose that $\mathcal{D} \subset \mathbb{R}^{2}$ is starlike with respect to the origin and that $A_{\mathcal{D}}(x)$ satisfies the asymptotic formula (1.1). If $\mathrm{RH}$ is true,

$$
B_{\mathcal{D}}(x)=\sum_{r: \alpha_{r}>\theta} \frac{c_{r}}{\zeta\left(2 \alpha_{r}\right)} x^{\alpha_{r}}+O\left(x^{\theta+\varepsilon}\right)
$$

for a large real variable $x$, arbitrary fixed $\varepsilon>0$, and

$$
\theta:=\frac{4-\alpha}{11-8 \alpha}
$$

Before going into technical details (which we postpone to Sections 2 and 3 ), we outline the essential ideas of the proof.

First of all, it will be convenient to consider the quantities

$$
A_{\mathcal{D}}^{*}(x):=\#\left\{\mathbf{m} \in \mathbb{Z}_{*}^{2}: Q(\mathbf{m}) \leq x\right\}
$$

and

$$
B_{\mathcal{D}}^{*}(x):=\#\left\{\mathbf{m}=\left(m_{1}, m_{2}\right) \in \mathbb{Z}_{*}^{2}: Q(\mathbf{m}) \leq x, \operatorname{gcd}\left(m_{1}, m_{2}\right)=1\right\},
$$

instead of $A_{\mathcal{D}}(x), B_{\mathcal{D}}(x)$. Since, for every $\delta>0$,

$$
A_{\mathcal{D}}(x) \leq A_{\mathcal{D}}^{*}(x) \leq A_{\mathcal{D}}(x+\delta), \quad B_{\mathcal{D}}^{*}(x-\delta) \leq B_{\mathcal{D}}(x) \leq B_{\mathcal{D}}^{*}(x),
$$

$A_{\mathcal{D}}^{*}(x)$ satisfies the asymptotic formula (1.1) as well, and the Theorem is immediate for $B_{\mathcal{D}}(x)$ if it has been proved for $B_{\mathcal{D}}^{*}(x)$.

Further, it is clear that

$$
Q_{1}:=\inf _{\mathbf{u} \in \mathbb{Z}_{*}^{2}} Q(\mathbf{u})>0
$$

Thus we may restrict the summation in (1.3) to $1 \leq m \leq \sqrt{x / Q_{1}}$, and obtain by splitting up

$$
B_{\mathcal{D}}^{*}(x)=\sum_{m \leq y} \mu(m) A_{\mathcal{D}}^{*}\left(\frac{x}{m^{2}}\right)+\sum_{m>y} \mu(m) A_{\mathcal{D}}^{*}\left(\frac{x}{m^{2}}\right)=: S_{1}+S_{2},
$$

where $y=y(x)<\sqrt{x / Q_{1}}$ is a parameter remaining at our disposition. By (1.1),

$$
S_{1}=\sum_{r=0}^{R} c_{r} x^{\alpha_{r}} \sum_{m \leq y} \frac{\mu(m)}{m^{2 \alpha_{r}}}+O\left(x^{\alpha} y^{1-2 \alpha}\right)
$$


Using the classic conditional bound (valid under $\mathrm{RH}$ )

$$
\sum_{m \leq Y} \mu(m) \ll Y^{1 / 2+\varepsilon^{\prime}} \quad\left(\varepsilon^{\prime}>0\right),
$$

summation by parts gives

$$
\sum_{m \leq y} \frac{\mu(m)}{m^{2 \alpha_{r}}}= \begin{cases}\frac{1}{\zeta\left(2 \alpha_{r}\right)}+O\left(y^{\frac{1}{2}-2 \alpha_{r}+\varepsilon^{\prime}}\right) & \text { if } \alpha_{r}>\frac{1}{4} \\ O\left(y^{\frac{1}{2}-2 \alpha_{r}+\varepsilon^{\prime}}\right) & \text { else. }\end{cases}
$$

Thus (1.7) may be rewritten in the form

$$
\begin{aligned}
S_{1}= & \sum_{r: \alpha_{r}>\frac{1}{3}(2+\alpha)} c_{r} x^{\alpha_{r}} \sum_{m \leq y} \frac{\mu(m)}{m^{2 \alpha_{r}}}+\sum_{r: \frac{1}{4}<\alpha_{r} \leq \frac{1}{3}(2+\alpha)} \frac{c_{r}}{\zeta\left(2 \alpha_{r}\right)} x^{\alpha_{r}} \\
& +\sum_{r: \alpha_{r} \leq \frac{1}{3}(2+\alpha)} O\left(x^{\alpha_{r}} y^{\frac{1}{2}-2 \alpha_{r}+\varepsilon^{\prime}}\right)+O\left(x^{\alpha} y^{1-2 \alpha}\right) .
\end{aligned}
$$

To deal with $S_{2}$, an obvious possibility is to use (1.8) one more time and to apply summation by parts repeatedly. Observing that $A_{\mathcal{D}}^{*}(w)$ is monotone and $\ll w$, one obtains

$$
S_{2} \ll x^{\varepsilon^{\prime}} y^{1 / 2} \frac{x}{y^{2}} .
$$

(See Moroz [15, formula (8)].)

The key step of the present paper is to improve this elementary estimate by a contour integration technique in the spirit of a classic paper due to Montgomery and Vaughan [14].

Proposition. If the Riemann Hypothesis is true,

$$
\begin{aligned}
& S_{2}=\sum_{m>y} \mu(m) A_{\mathcal{D}}^{*}\left(\frac{x}{m^{2}}\right)= \sum_{r: \alpha_{r}>\frac{1}{3}(2+\alpha)} c_{r} x^{\alpha_{r}} \sum_{m>y} \frac{\mu(m)}{m^{2 \alpha_{r}}} \\
&+O\left(x^{\alpha+\varepsilon^{\prime}}\right)+O\left(x^{\varepsilon^{\prime}} y^{1 / 2}\left(\frac{x}{y^{2}}\right)^{\frac{2+\alpha}{3}}\right) \quad\left(\varepsilon^{\prime}>0\right),
\end{aligned}
$$

for large real parameters $x$ and $y$ with $1 \leq y \ll x^{1 / 2}$.

We combine this result with (1.9) and note that the last $O$-terms in (1.9) and (1.10), respectively, are of the same order (apart from $\varepsilon$ 's) for

$$
y=x^{\frac{4(1-\alpha)}{11-8 \alpha}} .
$$

This choice of $y$ readily yields the assertion of our Theorem, since it is easily verified that, for $\alpha_{r} \leq \frac{1}{3}(2+\alpha)$,

$$
x^{\alpha_{r}} y^{\frac{1}{2}-2 \alpha_{r}} \ll x^{\theta} .
$$




\section{Some Lemmas.}

For $\operatorname{Re} s>1$, we define the zeta-function $Z_{\mathcal{D}}(s)$ of the set $\mathcal{D}$ by the absolutely convergent Dirichlet series

$$
Z_{\mathcal{D}}(s)=\sum_{\mathbf{m} \in \mathbb{Z}_{*}^{2}: Q(\mathbf{m})<\infty}(Q(\mathbf{m}))^{-s} .
$$

We further put, for real $y \geq 1$ and a complex variable $s$,

$$
f_{y}(s)=\frac{1}{\zeta(s)}-\sum_{m \leq y} \frac{\mu(m)}{m^{s}} .
$$

This is regular in every $s \in \mathbb{C}$ which is not a zero of the Riemann zetafunction.

Lemma 1. For a large real variable $x$, and any fixed $C \geq 5$,

$$
\begin{aligned}
S_{2} & =\sum_{m>y} \mu(m) A_{\mathcal{D}}^{*}\left(\frac{x}{m^{2}}\right) \\
& =\frac{1}{2 \pi i} \int_{3-i x^{C}}^{3+i x^{C}} Z_{\mathcal{D}}(s) f_{y}(2 s) \frac{x^{s}}{s} \mathrm{~d} s+O\left(x^{\alpha+\varepsilon}\right) \quad(\varepsilon>0),
\end{aligned}
$$

uniformly in $1 \leq y \ll \sqrt{x}$.

Proof. This clearly is a type of truncated Perron's formula. It is hard to find an explicit reference in the literature, although the argument runs on familiar lines:

Let us write the (positive and finite) values attained by $Q(\mathbf{m})$, as $\mathbf{m}$ runs through $\mathbb{Z}_{*}^{2}$, in form of a strictly increasing sequence $\left(\lambda_{k}\right)_{k \in \mathbb{N}}$. Put further

$$
\mu_{y}(m)= \begin{cases}\mu(m) & \text { if } m>y \\ 0 & \text { else }\end{cases}
$$

then it follows by the homogeneity of $Q$ that, for $\operatorname{Re} s>1$,

$$
Z_{\mathcal{D}}(s) f_{y}(2 s)=\sum_{\mathbf{n} \in \mathbb{Z}_{*}^{2}: Q(\mathbf{n})<\infty} \gamma(\mathbf{n})(Q(\mathbf{n}))^{-s}=\sum_{k=1}^{\infty} \eta_{k} \lambda_{k}^{-s},
$$

with

$$
\begin{aligned}
\gamma(\mathbf{n}) & :=\sum_{m \mid \mathbf{n}} \mu_{y}(m), \\
\eta_{k} & :=\sum_{\mathbf{n}:} \gamma(\mathbf{n})=\lambda_{k}
\end{aligned}
$$


Here $m \mid\left(n_{1}, n_{2}\right)$ means that $m \mid \operatorname{gcd}\left(n_{1}, n_{2}\right)$. For later reference, we note that, for any $\mathbf{n}=\left(n_{1}, n_{2}\right) \in \mathbb{Z}_{*}^{2}$ with $Q(\mathbf{n})<\infty$,

$$
\operatorname{gcd}\left(n_{1}, n_{2}\right) \ll Q(\mathbf{n}) \text {. }
$$

To realize this, let

$$
\mathbf{n}^{*}:=\left(\frac{n_{1}}{\operatorname{gcd}\left(n_{1}, n_{2}\right)}, \frac{n_{2}}{\operatorname{gcd}\left(n_{1}, n_{2}\right)}\right)
$$

then $\mathbf{n}^{*}, 2 \mathbf{n}^{*}, \ldots, \operatorname{gcd}\left(n_{1}, n_{2}\right) \mathbf{n}^{*}$ all belong to $\sqrt{2 Q(\mathbf{n})} \mathcal{D} \cap \mathbb{Z}_{*}^{2}$. Therefore, by $(1.1)$,

$$
\operatorname{gcd}\left(n_{1}, n_{2}\right) \leq A_{\mathcal{D}}(2 Q(\mathbf{n})) \ll Q(\mathbf{n}) .
$$

Furthermore,

$$
S_{2}=\sum_{m>y} \mu(m)\left(\sum_{Q(\mathbf{n}) \leq \frac{x}{m^{2}}} 1\right)=\sum_{m, \mathbf{n}: Q(m \mathbf{n}) \leq x} \mu_{y}(m)=\sum_{k: \lambda_{k} \leq x} \eta_{k} .
$$

It is well-known that, for $a>0, a \neq 1$, and $T$ sufficiently large,

$$
\frac{1}{2 \pi i} \int_{3-i T}^{3+i T} \frac{a^{s}}{s} \mathrm{~d} s=\left\{\begin{array}{l}
\chi(a)+O\left(\frac{a^{3}}{T|\log a|}\right), \\
O\left(a^{3}\right),
\end{array}\right.
$$

where $\chi$ is the characteristic function of the interval $] 1, \infty[$. Of this formula, $(*)$ may be found in Apostol [1, p. 243], while (**) is immediate by taking as a path of integration the boundary of the domain $\{s \in \mathbb{C}:|s| \leq T$, Re $s \leq 3\}$ if $a>1$, resp., of $\{s \in \mathbb{C}:|s| \leq T$, Re $s \geq 3\}$ if $a<1$ (cf. Prachar $[21$, p. 379]).

Therefore, by (2.2) and (2.4),

$$
\begin{aligned}
& \frac{1}{2 \pi i} \int_{3-i x^{C}}^{3+i x^{C}} Z_{\mathcal{D}}(s) f_{y}(2 s) \frac{x^{s}}{s} \mathrm{~d} s \\
& =S_{2}+\sum_{k:\left|\lambda_{k}-x\right| \geq 1} O\left(\frac{\left|\eta_{k}\right|}{\lambda_{k}^{3} x^{2}\left|\log \lambda_{k}-\log x\right|}\right)+\sum_{k:\left|\lambda_{k}-x\right|<1} O\left(\left|\eta_{k}\right|\right) .
\end{aligned}
$$

By the mean-value theorem,

$$
\left|\log \lambda_{k}-\log x\right|^{-1} \leq \frac{\max \left(\lambda_{k}, x\right)}{\left|\lambda_{k}-x\right|} \ll \frac{\lambda_{k} x}{\left|\lambda_{k}-x\right|},
$$


thus the first error term sum here is

$$
\ll \frac{1}{x} \sum_{k=1}^{\infty}\left|\eta_{k}\right| \lambda_{k}^{-2} \ll 1
$$

since the series in (2.2) converges absolutely for $\operatorname{Re} s>1$. Further,

$$
\left|\eta_{k}\right| \leq \sum_{\mathbf{n}: Q(\mathbf{n})=\lambda_{k}}|\gamma(\mathbf{n})| \ll \lambda_{k}^{\varepsilon^{\prime}} \sum_{\mathbf{n}: Q(\mathbf{n})=\lambda_{k}} 1,
$$

for any $\varepsilon^{\prime}>0$, in view of $(2.3)$ and the definition of $\gamma(\mathbf{n})$. Thus the second error term sum in (2.5) is

$$
\ll x^{\varepsilon^{\prime}} \sum_{\mathbf{n}:|Q(\mathbf{n})-x|<1} 1 \ll x^{\varepsilon^{\prime}}\left(A_{\mathcal{D}}^{*}(x+1)-A_{\mathcal{D}}^{*}(x-1)\right) \ll x^{\alpha+\varepsilon^{\prime}},
$$

in view of (1.1). This proves Lemma 1.

The key point to prove the Proposition will be to have at hand the following estimates for the growth of the complex function $Z_{\mathcal{D}}(s)$ in the vertical direction.

\section{Lemma 2.}

(i) For any $\sigma_{1}>\alpha$, there exists a positive real number $\omega<1$ such that

$$
Z_{\mathcal{D}}(\sigma+i t) \ll|t|^{\omega},
$$

uniformly in $\sigma \geq \sigma_{1},|t| \geq 1$.

(ii) For a real parameter $T \geq 4$, fixed $\varepsilon^{\prime}>0$, and any fixed $\beta$ with ${ }^{1}$ $\frac{2+\alpha}{3} \leq \beta<1$, it follows that

$$
\int_{T}^{2 T}\left|Z_{\mathcal{D}}(\beta+i t)\right| \mathrm{d} t \ll T^{1+\varepsilon^{\prime}}
$$

Proof. Let us rewrite (1.1) in the form

$$
P_{\mathcal{D}}^{*}(x):=A_{\mathcal{D}}^{*}(x)-\sum_{r=0}^{R} c_{r} x^{\alpha_{r}} \ll x^{\alpha} .
$$

Let further $X$ denote a positive real number which is not attained by $Q(\mathbf{n})$ as $\mathbf{n}$ runs through $\mathbb{Z}_{*}^{2}$. Using Stieltjes integral calculus, we conclude that,

\footnotetext{
${ }^{1}$ For $\beta \geq 1$, the estimate is trivial and not needed later.
} 
for $\operatorname{Re} s>1$,

$$
\begin{aligned}
& Z_{\mathcal{D}}(s)-\sum_{0<Q(\mathbf{n}) \leq X}(Q(\mathbf{n}))^{-s}=\int_{X}^{\infty} w^{-s} \mathrm{~d}\left(\sum_{r=0}^{R} c_{r} w^{\alpha_{r}}+P_{\mathcal{D}}^{*}(w)\right) \\
& =\sum_{r=0}^{R} c_{r} \alpha_{r} \int_{X}^{\infty} w^{-s+\alpha_{r}-1} \mathrm{~d} w+\int_{X}^{\infty} w^{-s} \mathrm{~d} P_{\mathcal{D}}^{*}(w) \\
& =\sum_{r=0}^{R} c_{r} \frac{\alpha_{r}}{s-\alpha_{r}} X^{\alpha_{r}-s}-X^{-s} P_{\mathcal{D}}^{*}(X)+s \int_{X}^{\infty} w^{-s-1} P_{\mathcal{D}}^{*}(w) \mathrm{d} w .
\end{aligned}
$$

In this identity we choose $0<X<Q_{1}$ and let $X \rightarrow Q_{1}-$ to obtain

$$
Z_{\mathcal{D}}(s)=\sum_{r=0}^{R} c_{r} \frac{s}{s-\alpha_{r}} Q_{1}^{\alpha_{r}-s}+s \int_{Q_{1}}^{\infty} w^{-s-1} P_{\mathcal{D}}^{*}(w) \mathrm{d} w .
$$

In view of (2.6), this provides a meromorphic continuation of $Z_{\mathcal{D}}(s)$ to the half-plane $\operatorname{Re} s>\alpha$, with simple poles at $s=\alpha_{r}, r=0, \ldots, R$. At the same time, (2.8) shows that

$$
Z_{\mathcal{D}}(\sigma+i t) \ll|t|,
$$

uniformly in $\sigma \geq \sigma_{0},|t| \geq 1$, where $\sigma_{0}>\alpha$ is arbitrary but fixed. Since, by absolute convergence, $Z_{\mathcal{D}}(\sigma+i t)$ is uniformly bounded in every half-plane $\sigma \geq \sigma_{2}>1$, a Phragmén-Lindelöf argument ${ }^{2}$ establishes part (i) of Lemma 2 , if we put $\sigma_{0}=\frac{1}{2}\left(\alpha+\sigma_{1}\right)$ for arbitrary given $\sigma_{1}>\alpha$.

To show (ii), we apply the identity derived in (2.7) one more time, with

$$
\begin{gathered}
T^{\xi} \leq X \leq 2 T^{\xi}, \quad \xi:=\frac{3}{2(1-\alpha)} \\
s=\beta+i t, \quad T \leq t \leq 2 T .
\end{gathered}
$$

This is clearly justified by analytic continuation. We obtain

$$
Z_{\mathcal{D}}(\beta+i t)=S_{X}(t)+O\left(T^{-1} X^{1-\beta}\right)+O\left(T X^{-\beta+\alpha}\right)
$$

with

$$
S_{X}(t):=\sum_{\mathbf{m} \in \mathbb{Z}_{*}^{2}: Q(\mathbf{m}) \leq X}(Q(\mathbf{m}))^{-\beta-i t} .
$$

\footnotetext{
${ }^{2}$ For a classic reference, see Landau [13, p. 229].
} 
Integration over $T \leq t \leq 2 T$ gives

$$
\int_{T}^{2 T}\left|Z_{\mathcal{D}}(\beta+i t)\right| \mathrm{d} t \ll \int_{T}^{2 T}\left|S_{X}(t)\right| \mathrm{d} t+O\left(X^{1-\beta}\right)+O\left(T^{2} X^{-\beta+\alpha}\right) .
$$

By Cauchy's inequality ${ }^{3}$,

$$
\begin{aligned}
\left(\int_{T}^{2 T}\left|S_{X}(t)\right| \mathrm{d} t\right)^{2} & \ll T \int_{T}^{2 T}\left|S_{X}(t)\right|^{2} \mathrm{~d} t \\
& \ll T \sum_{Q(\mathbf{m}) \leq Q(\mathbf{n}) \leq X}(Q(\mathbf{m}) Q(\mathbf{n}))^{-\beta}\left|\int_{T}^{2 T}\left(\frac{Q(\mathbf{n})}{Q(\mathbf{m})}\right)^{i t} \mathrm{~d} t\right|
\end{aligned}
$$

For $Q(\mathbf{m})<Q(\mathbf{n})$, the integrals in this sum can be estimated by

$$
\begin{aligned}
\left|\int_{T}^{2 T} \exp (i t(\log Q(\mathbf{n})-\log Q(\mathbf{m}))) \mathrm{d} t\right| & \leq \frac{2}{\log Q(\mathbf{n})-\log Q(\mathbf{m})} \\
& \leq \frac{2 Q(\mathbf{n})}{Q(\mathbf{n})-Q(\mathbf{m})}
\end{aligned}
$$

Along with the trivial bound, this gives

$$
\begin{aligned}
& \left(\int_{T}^{2 T}\left|S_{X}(t)\right| \mathrm{d} t\right)^{2} \ll T \sum_{Q(\mathbf{n}) \leq X}(Q(\mathbf{n}))^{-\beta} \\
\times & \left(\sum_{\mathbf{m}:}(Q(\mathbf{m}) \leq Q(\mathbf{n})\right. \\
& \left.(\operatorname{m}))^{-\beta}\left(\max \left(\frac{1}{T}, \frac{Q(\mathbf{n})-Q(\mathbf{m})}{Q(\mathbf{n})}\right)\right)^{-1}\right) .
\end{aligned}
$$

We now keep $\mathbf{n} \in \mathbb{Z}_{*}^{2}$ fixed for the moment and split up the inner sum over $\mathbf{m}$ : For that purpose, we define a sequence $\left(\delta_{j}\right)_{j=0}^{J}$ by $\delta_{j}=2^{j} Q(\mathbf{n}) T^{-1}$, with $J$ such that $\frac{1}{8} Q(\mathbf{n})<\delta_{J} \leq \frac{1}{4} Q(\mathbf{n})$. We distinguish three cases according to the relative size of $Q(\mathbf{n})-Q(\mathbf{m})$.

First of all (Case 1),

$$
\begin{aligned}
Q(\mathbf{n})-Q(\mathbf{m})<\delta_{0} & \Longleftrightarrow \frac{1}{T}>\frac{Q(\mathbf{n})-Q(\mathbf{m})}{Q(\mathbf{n})} \\
& \Longleftrightarrow Q(\mathbf{m})>Q(\mathbf{n})\left(1-\frac{1}{T}\right)
\end{aligned}
$$

\footnotetext{
${ }^{3}$ Here and in what follows, $\mathbf{m}$ and $\mathbf{n}$ denote elements of $\mathbb{Z}_{*}^{2}$.
} 
thus the contribution of these $\mathbf{m}$ to the inner sum in (2.12) is

$$
\begin{aligned}
& \ll(Q(\mathbf{n}))^{-\beta} T\left(A_{\mathcal{D}}^{*}(Q(\mathbf{n}))-A_{\mathcal{D}}^{*}\left(Q(\mathbf{n})\left(1-\frac{1}{T}\right)\right)\right) \\
& \ll(Q(\mathbf{n}))^{1-\beta}+T(Q(\mathbf{n}))^{-\beta+\alpha} .
\end{aligned}
$$

Further (Case 2), for $0 \leq j \leq J$,

$$
Q(\mathbf{n})-Q(\mathbf{m}) \in\left[\delta_{j}, 2 \delta_{j}\left[\quad \Longleftrightarrow \quad Q(\mathbf{n})-2 \delta_{j}<Q(\mathbf{m}) \leq Q(\mathbf{n})-\delta_{j},\right.\right.
$$

thus the corresponding portion of the inner sum in (2.12) is

$$
\begin{aligned}
& \ll \frac{(Q(\mathbf{n}))^{1-\beta}}{\delta_{j}}\left(A_{\mathcal{D}}^{*}\left(Q(\mathbf{n})-\delta_{j}\right)-A_{\mathcal{D}}^{*}\left(Q(\mathbf{n})-2 \delta_{j}\right)\right) \\
& \ll(Q(\mathbf{n}))^{1-\beta}+\delta_{j}^{-1}(Q(\mathbf{n}))^{1-\beta+\alpha} .
\end{aligned}
$$

Summing this over $j=0, \ldots, J$ gives

$$
O\left(J(Q(\mathbf{n}))^{1-\beta}\right)+O\left(\delta_{0}^{-1}(Q(\mathbf{n}))^{1-\beta+\alpha}\right) \ll T^{\varepsilon}(Q(\mathbf{n}))^{1-\beta}+T(Q(\mathbf{n}))^{\alpha-\beta} .
$$

Finally (Case 3 ), the portion of the inner sum in (2.12) corresponding to the m's with $Q(\mathbf{n})-Q(\mathbf{m}) \geq 2 \delta_{J}$ is

$$
\ll \sum_{\mathbf{m}: Q(\mathbf{m}) \leq Q(\mathbf{n})}(Q(\mathbf{m}))^{-\beta}=\int_{\frac{1}{2}}^{Q(\mathbf{n})} u^{-\beta} \mathrm{d} A_{\mathcal{D}}^{*}(u) \ll(Q(\mathbf{n}))^{1-\beta} .
$$

We now combine the upper bounds (2.13), (2.14), and (2.15), and use them in (2.12) to conclude that

$$
\begin{aligned}
\left(\int_{T}^{2 T}\left|S_{X}(t)\right| \mathrm{d} t\right)^{2} & \ll T \sum_{Q(\mathbf{n}) \leq X}(Q(\mathbf{n}))^{-\beta}\left(T^{\varepsilon}(Q(\mathbf{n}))^{1-\beta}+T(Q(\mathbf{n}))^{\alpha-\beta}\right) \\
& =T^{1+\varepsilon} \int_{\frac{1}{2}}^{X} w^{1-2 \beta} \mathrm{d} A_{\mathcal{D}}^{*}(w)+T^{2} \ll T^{1+\varepsilon} X^{2-2 \beta}+T^{2} .
\end{aligned}
$$

Combining this with (2.11), we obtain

$$
\int_{T}^{2 T}\left|Z_{\mathcal{D}}(\beta+i t)\right| \mathrm{d} t \ll T^{1 / 2+\varepsilon} X^{1-\beta}+T+X^{1-\beta}+T^{2} X^{-\beta+\alpha} .
$$

It is easy to see that the choice of $X$ according to (2.9) is optimal, and that the bound obtained is $\ll T^{1+\varepsilon}$ for $\beta \geq \frac{2+\alpha}{3}$. Thus the proof of Lemma 2 is complete. 
Lemma 3. If $\mathrm{RH}$ is true, the function $f_{y}(s)$ defined in (2.1) satisfies

$$
f_{y}(\sigma+i t) \ll y^{\frac{1}{2}-\sigma+\varepsilon^{\prime}}\left(|t|^{\varepsilon^{\prime}}+1\right) \quad\left(\varepsilon^{\prime}>0 \text { fixed }\right),
$$

uniformly in $\sigma_{1} \leq \sigma \leq \sigma_{2}, y \geq 1$, for arbitrary $\sigma_{2}>\sigma_{1}>\frac{1}{2}$.

Proof. This key lemma of the Montgomery-Vaughan method is meanwhile well-known. See, e.g., Nowak and Schmeier [20], or Baker [2, Lemma 1].

\section{Proof of the Proposition.}

We put

$$
\beta:=\frac{2+\alpha}{3}+\varepsilon^{\prime \prime}
$$

with $\varepsilon^{\prime \prime} \geq 0$ as small as we please, such that ${ }^{4}$

$$
\beta \notin\left\{\alpha_{0}, \ldots, \alpha_{R}\right\} \text {. }
$$

We start from Lemma 1 and shift the line of integration to $\operatorname{Re} s=\beta$, applying the residue theorem. In view of clause (i) of Lemma 2 and Lemma 3, the horizontal segments contribute

$$
\ll x^{3-C} \int_{\beta}^{3}\left|Z_{\mathcal{D}}\left(\sigma+i x^{C}\right) f_{y}\left(2 \sigma+2 i x^{C}\right)\right| \mathrm{d} \sigma \ll x^{3-C+C\left(\omega+\varepsilon^{\prime}\right)} \ll 1
$$

for $C$ sufficiently large. Furthermore, by clause (ii) of Lemma 2 and Lemma 3,

$$
\begin{aligned}
& \int_{\beta-i x^{C}}^{\beta+i x^{C}} Z_{\mathcal{D}}(s) f_{y}(2 s) x^{s} \frac{\mathrm{d} s}{s} \\
& \ll x^{\beta} y^{\frac{1}{2}-2 \beta+\varepsilon^{\prime}}\left(1+\sum_{T=2^{-j} x^{C}, j=1,2, \ldots} T^{\varepsilon^{\prime}-1} \int_{T}^{2 T}\left|Z_{\mathcal{D}}(\beta+i t)\right| \mathrm{d} t\right) \\
& \ll x^{\beta+2 C \varepsilon^{\prime}} y^{\frac{1}{2}-2 \beta+\varepsilon^{\prime}} .
\end{aligned}
$$

Collecting results, we arrive at

$$
S_{2}=\sum_{r: \alpha_{r}>\beta} \operatorname{Res}_{s=\alpha_{r}}\left(Z_{\mathcal{D}}(s) f_{y}(2 s) \frac{x^{s}}{s}\right)+O\left(x^{\alpha+\varepsilon^{\prime}}\right)+O\left(x^{\varepsilon} y^{1 / 2}\left(\frac{x}{y^{2}}\right)^{\frac{2+\alpha}{3}}\right) \text {. }
$$

Since, by (2.8),

$$
\operatorname{Res}_{s=\alpha_{r}}\left(Z_{\mathcal{D}}(s) f_{y}(2 s) \frac{x^{s}}{s}\right)=c_{r} x^{\alpha_{r}} \sum_{m>y} \frac{\mu(m)}{m^{2 \alpha_{r}}}
$$

for $\alpha_{r}>\beta$, this completes the proof of the Proposition and thereby that of our Theorem.

\footnotetext{
${ }^{4} \varepsilon^{\prime \prime}$ is only needed to deal with the case that $\frac{2+\alpha}{3}$ is equal to one of $\alpha_{0}, \ldots, \alpha_{R}$.
} 


\section{Some applications to special problems.}

4.1. Convex domains with nonzero curvature of the boundary. The most "generic" example is probably a convex planar domain $\mathcal{D}$ whose boundary $\partial \mathcal{D}$ is sufficiently $\operatorname{smooth}^{5}$ and has nonzero curvature throughout. We further suppose that the origin is an inner point of $\mathcal{D}$. Under these conditions, a very deep and rather recent result of Huxley [6] says that

$$
A_{\mathcal{D}}(x)=\operatorname{area}(\mathcal{D}) x+O\left(x^{\frac{23}{73}}(\log x)^{\frac{315}{146}}\right) .
$$

Using this with our Theorem, we obtain for the number of primitive lattice points in $\sqrt{x} \mathcal{D}$ (conditionally under $\mathrm{RH}$ ),

$$
B_{\mathcal{D}}(x)=\frac{6}{\pi^{2}} \operatorname{area}(\mathcal{D}) x+O\left(x^{\frac{269}{619}+\varepsilon}\right) .
$$

However, for this special problem, Huxley and the author [7] have established the better error term $O\left(x^{\frac{5}{12}+\varepsilon}\right)$. (Numerically, $\frac{269}{619}=0.434571 \ldots$, while $\left.\frac{5}{12}=0.416666 \ldots\right)$ This result does not depend on (4.1), but was derived using the mean-square bound

$$
\int_{0}^{x}\left(A_{\mathcal{D}}(u)-\operatorname{area}(\mathcal{D}) u\right)^{2} \mathrm{~d} u \ll x^{3 / 2} .
$$

For the case that $\mathcal{D}$ is the unit disk (or any origin-centered rational ellipse), a recent idea of Baker [2] can be modified to prove (under $\mathrm{RH}$ ) that

$$
B_{\mathcal{D}}(x)=\frac{6}{\pi} x+O\left(x^{\frac{3}{8}+\varepsilon}\right) .
$$

(See also [7] for a bit more details.)

4.2. Sums and differences of relative prime $k$-th powers. For a fixed natural number $k \geq 3$, we ask for the average order of the arithmetic functions $r_{k}^{+}(n), r_{k}^{-}(n)$, and $\rho_{k}^{+}(n), \rho_{k}^{-}(n)$, which are defined, respectively, by

$$
\begin{aligned}
& r_{k}^{ \pm}(n):=\left\{(u, v) \in \mathbb{Z}^{2}:|u|^{k} \pm|v|^{k}=n\right\}, \\
& \rho_{k}^{ \pm}(n):=\left\{(u, v) \in \mathbb{Z}^{2}:|u|^{k} \pm|v|^{k}=n, \operatorname{gcd}(u, v)=1\right\} .
\end{aligned}
$$

From a geometric viewpoint, these functions are associated with the starlike planar domains

$$
\mathcal{D}^{ \pm}:=\left\{(u, v) \in \mathbb{R}^{2}: 0<|u|^{k} \pm|v|^{k} \leq 1\right\} .
$$

\footnotetext{
${ }^{5}$ To be precise, it suffices that the curvature of $\partial \mathcal{D}$, as a function of the arclength, is twice continuously differentiable.
} 
It is known from classic results of Krätzel [9], [10], [11] that

$$
\sum_{1 \leq n \leq T} r_{k}^{ \pm}(n)=A_{\mathcal{D}^{ \pm}}\left(T^{2 / k}\right)=c_{0}^{ \pm}(k) T^{2 / k}+c_{1}^{ \pm}(k) T^{1 /(k-1)}+O\left(T^{\frac{1}{k}-\frac{1}{k^{2}}}\right)
$$

with

$$
\begin{aligned}
c_{0}^{+}(k) & =\frac{2 \Gamma^{2}\left(\frac{1}{k}\right)}{k \Gamma\left(\frac{2}{k}\right)}, & c_{0}^{-}(k) & =\frac{\Gamma^{2}\left(\frac{1}{k}\right)}{k \cos \left(\frac{\pi}{k}\right) \Gamma\left(\frac{2}{k}\right)}, \\
c_{1}^{+}(k) & =0, & c_{1}^{-}(k) & =4 \zeta\left(\frac{1}{k-1}\right) k^{-1 /(k-1)} .
\end{aligned}
$$

Our Theorem readily implies (provided that $\mathrm{RH}$ is true)

$$
\begin{aligned}
\sum_{1 \leq n \leq T} \rho_{k}^{ \pm}(n) & =B_{\mathcal{D}^{ \pm}}\left(T^{2 / k}\right) \\
& =\frac{6}{\pi^{2}} c_{0}^{ \pm}(k) T^{2 / k}+\frac{c_{1}^{ \pm}(k)}{\zeta\left(\frac{k}{k-1}\right)} T^{1 /(k-1)}+O\left(T^{\frac{7 k+1}{k(7 k+4)}+\varepsilon}\right) .
\end{aligned}
$$

Again the estimate can be improved slightly, making use of more precise representations of the error term in (4.2) (see [19]).

4.3. Primitive Pythagorean triangles. Let us define as a primitive Pythagorean triangle any triple of natural numbers $(u, v, w)$ satisfying

$$
u^{2}+v^{2}=w^{2}, \quad u \leq v, \quad \operatorname{gcd}(u, v, w)=1 .
$$

For a large real parameter $\mathcal{A}$, let $p(\mathcal{A})$ denote the number of primitive Pythagorean triangles with area less than $\mathcal{A}$. The problem to establish an asymptotic formula for $p(\mathcal{A})$ has been attacked by Lambek and Moser [12], Wild [22], Duttlinger and Schwarz [4], Müller, Nowak and Menzer [17], and Müller and Nowak [16]. According to Lambek and Moser [12], it is known that

$$
p(\mathcal{A})=\sum_{k=0}^{\infty}(-1)^{k} B_{\mathcal{D}}\left(\sqrt{\mathcal{A}} 2^{-k}\right)
$$

where

$$
\mathcal{D}:=\left\{(u, v) \in \mathbb{R}^{2}: u v\left(u^{2}-v^{2}\right)<1,0<v<u\right\} .
$$

In $[16]$ it has been shown that

$$
A_{\mathcal{D}}(x)=c_{0} x+c_{1} x^{2 / 3}+O\left(x^{7 / 22}(\log x)^{45 / 22}\right),
$$


with

$$
c_{0}=\frac{\Gamma^{2}\left(\frac{1}{4}\right)}{4 \sqrt{2 \pi}}, \quad c_{1}=-\left|\zeta\left(\frac{1}{3}\right)\right|\left(1+2^{-1 / 3}\right)
$$

Applying the most recent version of Huxley's lattice point theorems [6], it is straightforward to sharpen this error term to $O\left(x^{\frac{23}{73}}(\log x)^{\frac{315}{146}}\right)$. Using this with our Theorem and (4.3), one obtains (conditionally under $\mathrm{RH}$ )

$$
p(\mathcal{A})=c_{0}^{*} \mathcal{A}^{1 / 2}+c_{1}^{*} \mathcal{A}^{1 / 3}+O\left(\mathcal{A}^{\frac{269}{1238}+\varepsilon}\right)
$$

with

$$
c_{0}^{*}=\frac{\Gamma^{2}\left(\frac{1}{4}\right)}{\sqrt{2 \pi^{5}}}, \quad c_{1}^{*}=-\frac{\left|\zeta\left(\frac{1}{3}\right)\right|\left(1+2^{-1 / 3}\right)}{\zeta\left(\frac{4}{3}\right)\left(1+4^{-1 / 3}\right)},
$$

which improves upon all earlier results of this kind. (Numerically, $\frac{269}{1238}=$ $0.217285 \ldots$, while the best exponent in the error term known before was $\left.\frac{37}{164}=0.225609 \ldots\right)$

4.4. Primitive lattice points in special asteroid-shaped domains. As a last somewhat "exotic" example we consider starlike sets

$$
\mathcal{D}_{a}:=\left\{(u, v) \in \mathbb{R}^{2}:|u|^{a}+|v|^{a} \leq 1\right\}
$$

where $a$ is a fixed real number with $0<a<1$. It was known already to van der Corput [3] that

$$
A_{\mathcal{D}_{a}}(x)=\operatorname{area}\left(\mathcal{D}_{a}\right) x+\sum_{1 \leq r<\frac{1}{a}(1-2 \lambda)} c_{r}(a) x^{(1-a r) / 2}+O\left(x^{\lambda}\right),
$$

with

$$
c_{r}(a)=\frac{8(-1)^{r} \zeta(-a r) \Gamma\left(1+\frac{1}{a}\right)}{r ! \Gamma\left(1+\frac{1}{a}-r\right)},
$$

for $\lambda=\frac{1}{3}$. (Cf. also [18] for a generalization.) Appealing again to Huxley's work [6], this can be readily established for every $\lambda>\frac{23}{73}$. Thus our Theorem implies that (if $\mathrm{RH}$ is true)

$$
B_{\mathcal{D}_{a}}(x)=\frac{6}{\pi^{2}} \text { area }\left(\mathcal{D}_{a}\right) x+\sum_{1 \leq r<\frac{81}{619 a}} \frac{c_{r}(a)}{\zeta(1-a r)} x^{(1-a r) / 2}+O\left(x^{\frac{269}{619}+\varepsilon}\right) .
$$




\section{References}

[1] T.M. Apostol, Introduction to analytic number theory, Springer, New York - Heidelberg - Berlin, 1976.

[2] R.C. Baker, The square-free divisor problem, Quart. J. Math. Oxford, 45 (1994), 269-277.

[3] J.G. van der Corput, Over roosterpunkten in het plate vlak, (Dutch), Thesis Groningen, 1919.

[4] J. Duttlinger and W. Schwarz, Über die Verteilung der Pythagoräischen Dreiecke, Colloquium Math., 43 (1980), 365-372.

[5] D. Hensley, The number of lattice points within a contour and visible from the origin, Pacific J. Math., 166 (1994), 295-304.

[6] M.N. Huxley, Exponential sums and lattice points II, Proc. London Math. Soc., 66 (1993), 279-301.

[7] M.N. Huxley and W.G. Nowak, Primitive lattice points in convex planar domains, Acta Arithm., 76 (1996), 271-283.

[8] A. Ivić, The Riemann Zeta-function, J. Wiley \& Sons, New York and Chichester, 1985.

[9] E. Krätzel, Bemerkungen zu einem Gitterpunktproblem, Math. Ann., 179 (1969), 90-96.

[10] _ Mittlere Darstellungen natürlicher Zahlen als Differenz zweier $k$-ter Potenzen, Acta Arithm., 16 (1969), 111-121.

[11] L Lattice Points, Dt. Verl. d. Wiss., Berlin, 1988.

[12] J. Lambek and L. Moser, On the distribution of Pythagorean triangles, Pacific J. Math., 5 (1955), 73-83.

[13] E. Landau, Ausgewählte Abhandlungen zur Gitterpunktlehre, ed. by A. Walfisz, Dt. Verl. d. Wiss., Berlin, 1962.

[14] H.L. Montgomery and R.C. Vaughan, The distribution of squarefree numbers, in "Recent Progress in Analytic Number Theory", Proc. Durham Symp. 1979, Vol. I, (H. Halberstam and C. Hooley, Editors), Academic Press, London, (1981), 247-256.

[15] B.Z. Moroz, On the number of primitive lattice points in plane domains, Monatsh. Math., 99 (1985), 37-43.

[16] W. Müller and W.G. Nowak, Lattice points in planar domains: Applications of Huxley's "Discrete Hardy-Littlewood method", in: "Number theoretic analysis ", Seminar Vienna 1988-89, Springer Lecture Notes, 1452 (eds. E. Hlawka and R. F. Tichy), (1990), 139-164.

[17] W. Müller, W.G. Nowak and H. Menzer, On the number of primitive Pythagorean triangles, Ann. Sci. Math., Québec, 12 (1988), 263-273.

[18] W.G. Nowak, A non-convex generalization of the circle problem, J. Reine Angew. Math., 314 (1980), 136-145.

[19] W.G. Nowak, Sums and differences of two relative prime cubes, II, Proc. Czech and Slovake Number Theory Conference, 1995, in press.

[20] W.G. Nowak and M. Schmeier, Conditional asymptotic formulae for a class of arithmetic functions, Proc. Amer. Math. Soc., 103 (1988), 713-717. 
[21] K. Prachar, Primzahlverteilung, Springer Verlag, Berlin-Heidelberg-New York, 1957.

[22] R.E. Wild, On the number of primitive Pythagorean triangles with area less than $n$, Pacific J. Math., 5 (1955), 85-91.

Received September 20, 1995 and revised December 19, 1995. This article is part of a research project supported by the Austrian Science Foundation (NR. P 9892-PHY).

UNIVERSITÄT FÜR BODENKULTUR

A-1180 WiEn, Austria

E-mail address: nowak@mail.boku.ac.at 